\title{
INFLUENCE OF AN IMMUNOLOGICAL PRECIPITATE ON D.C. AND A.C. BEHAVIOUR OF AN ISFET
}

\author{
R. B. M. SCHASFOORT ${ }^{\dagger} *$, G. J. STREEKSTRA ${ }^{\dagger}$, P. BERGVELD*, \\ R. P. H. KOOYMAN ${ }^{\dagger}$ and J. GREVE ${ }^{\dagger}$
}

Department of Applied Physics ${ }^{\dagger}$ and Department of Electrical Engineering*, University of Twente, P.O. Box 217, 7500 AE Enschede (The Netherlands)

(Received August 10, 1987 ; in revised form May 10, 1988; accepted July 19, 1988)

\section{Abstract}

We propose to detect the charge redistribution of an antibody-antigen complex (a precipitate), formed in a layer on top of an ISFET, by means of a.c. measurements. In fact an increased specific conductivity of the precipitate is detected, caused by the amount of counterions, which is related to the fixed protein charge in the precipitate. We observed that an HSA-antiHSA immune complex, deposited onto an ISFET after immersing in an electrolyte, resulted in a cut-off frequency $(-3 \mathrm{~dB})$ that was considerably higher than that of a bare ISFET. The cut-off frequency decreased due to outdiffusion of ions until an equilibrium value was reached, but this was still higher than the typical value for the original bare ISFET. It was concluded that in the equilibrium state, the increased conductivity of the protein layer was the result of the counterions, which screen the fixed charges in the precipitate. Ionic strength and $\mathrm{pH}$ cause important changes in the effects. The measurement of the cut-off frequency may be used to determine the isoelectric point of a protein complex.

\section{Introduction}

The antibody-antigen interaction is one of the most specific biochemical reactions. Modern immunoassays are based on these reactions and enable picomoles or less of antigen to be detected. However, immunoassays such as RIA and ELISA detect label molecules and not the direct immunological reaction. It is obvious that it is more desirable to use the specific immunological reaction for direct potentiometric sensing without the use of labelled molecules.

In 1976 Schenck [1] proposed to detect the immunological reaction by means of an immunological field effect transistor (IMFET). He suggested that the formation of an antibody-antigen complex on the gate of an ISFET would lead to a detectable change in charge distribution. 
Janata and Huber [2] reported in 1980 that an IMFET is feasible if specific conditions are fulfilled. Nevertheless, attempts by different research groups to develop a reproducibly-operating IMFET have failed so far. In 1986 Janata [3] ascribed this failure to the fact that the diffuse charges of proteins are always screened by small counterions, resulting macroscopically in uncharged layers.

In this paper we will investigate the effect immunological molecules have on the ISFET characteristics. Therefore we have to focus in more detail on the operation of an ISFET with a deposited protein layer.

The ISFET, schematically shown in Fig. 1(a), has a drain current in the non-saturated mode of operation given by [4]

$$
I_{\mathrm{d}}=\mu C_{\mathrm{ox}} \frac{W}{L}\left[\left(V_{\mathrm{g}}-V_{\mathrm{t}}\right) V_{\mathrm{d}}-1 / 2 V_{\mathrm{d}}^{2}\right]
$$

where $\mu$ is the electron mobility, $C_{\text {ox }}$ the insulator capacitance per unit area, $L$ the length of the channel between source and drain, $W$ the channel width, $V_{g}$ the gate-source voltage and $V_{t}$ the threshold voltage. The threshold voltage represents all the effects of substrate depletion charge, interface states and fixed charges in the oxide. In practical measurements, the drain current is measured by applying a drain-source voltage $V_{d}$ to the device. With respect to a.c. measurements,

$$
\delta I_{\mathrm{d}} / \delta V_{\mathrm{g}}=i_{\mathrm{d}} / v_{\mathrm{g}}=\mu C_{\mathrm{ox}} \frac{W}{L} V_{\mathrm{d}}
$$

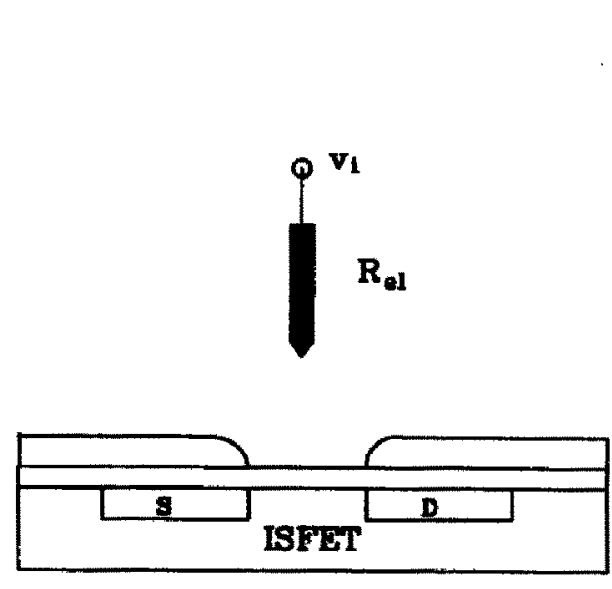

(a)

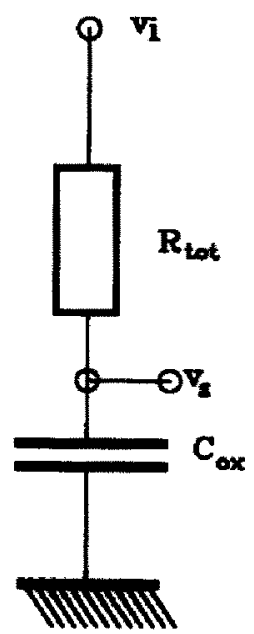

(b)

Fig. 1.(a) Schematic representation of an ISFET with reference electrode. (b) Simplified electrical model of the input circuit of (a).

Here $v_{\mathrm{s}}$ is the a.c. gate-source potential, which may differ from the a.c. potential $v_{1}$ applied to the reference electrode. The ratio $v_{g} / v_{1}$ represents the transfer function of the simple electronic model shown in Fig. 1(b), where $R_{\text {tot }}$ is the overall resistance of the bulk solution and the reference electrode:. 
Now the measuring system can be considered by a low-pass filter with cut-off frequency $f_{\mathrm{c}}(-3 \mathrm{~dB})$ :

$f_{\mathrm{c}}=\frac{1}{2 \pi R_{\mathrm{tot}} C_{\mathrm{ox}}}$

As will be shown here, proteins deposited on the gate surface can affect the cut-off frequency. We also investigated which factors determine the transfer characteristic.

\section{A qualitative model}

The cut-off frequency of the circuit, as shown in Fig. 1(b), can be influenced by changes in the series resistances $R_{\text {tot }}$, the ISFET input capacitance $C_{\mathrm{ox}}$ or both. The largest contribution to the total resistance between the ISFET surface and the reference electrode is due to the layer that lies closest to the gate of the ISFET. This implies that resistance changes in the relatively small volume around the gate may result in a detectable change of the total resistance. Capacitative effects can only be expected when the input capacitance is decreased due to a series capacitance that is not shortcircuited by a conductive fluid. Here we assume, however, a pure resistive contribution of adsorbed protein molecules on the transfer characteristic. Later we will discuss this assertion. The geometry of the actual measuring site with respect to resistive effects is schematically shown in Fig. 2(a). The solution resistance of the electrolyte is assumed to consist of local resistances $R_{\mathrm{s}}$ between equipotential surfaces $\left(A_{i}\right)$, at distance $l$. The total resistance $\boldsymbol{R}_{\text {tot }}$ can now be expressed as

$$
R_{\mathrm{tot}}=\sigma^{-1} \sum_{i=1}^{\infty} \frac{l}{A_{i}}+R_{\mathrm{el}}
$$

where

$\sigma=$ specific conductivity of the electrolyte,

$l=$ distance between two equipotential surfaces,

$A_{i}=$ equipotential surface area of layer $i$,

$R_{\text {el }}=$ resistance of reference electrode.

Because of the geometry of the electrolyte shells, $R_{\mathrm{s1}}>R_{\mathrm{s2}}>R_{\mathrm{s3}}>$ etc.

In Fig. 2(b) a simple electrical model of Fig. 2(a) is presented in which $R_{\mathrm{s} 1}$ is assumed to be variable, depending on the presence or absence of a protein precipitate. Inside the protein precipitate we assume a different specific conductivity $\sigma_{\mathrm{p}}$ so that eqn. (4) is modified to

$R_{\mathrm{tot}}=\sigma_{\mathrm{p}}^{-1} \frac{l}{A_{\mathrm{p}}}+\sigma^{-1} \sum_{i=2}^{\infty} \frac{l}{A_{i}}+R_{\mathrm{el}}$

Because $l / A_{i}$ has a very high value near the gate area, the protein layer has a relatively large effect upon the total resistance. From this qualitative 


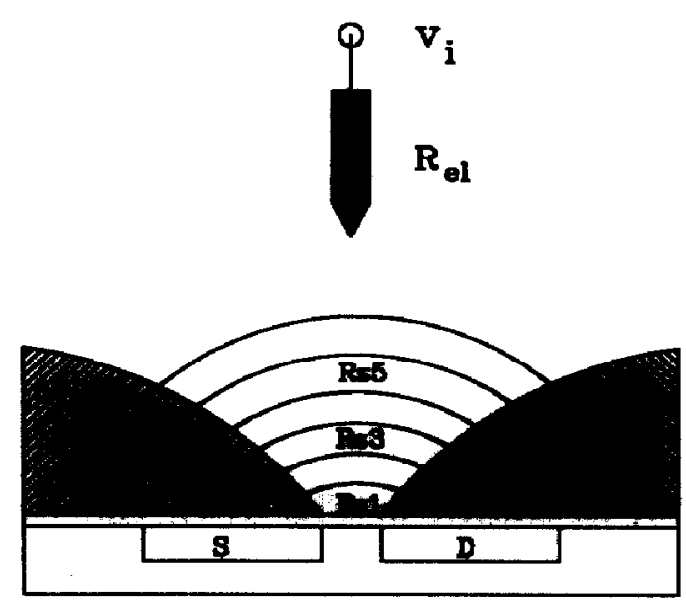

(a)

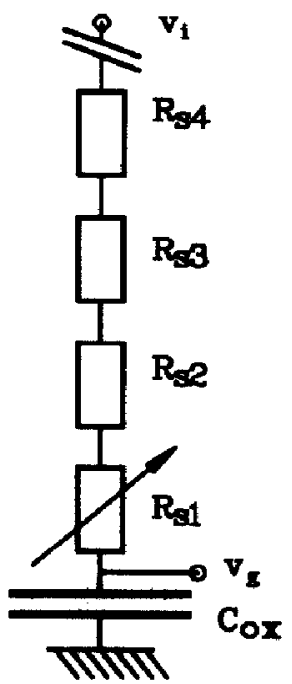

(b)

Fig. 2. (a) Schematic representation of electrolyte resiatances on top of an ISFET. (b) Simplified electrical model input circuit of (a).

model it may be expected that the a.c. behaviour of a bare ISFET with respect to an ISFET with a protein precipitate will be different due to a frequency-dependent voltage division between $R_{\text {tot }}$ and $C_{\mathrm{ox}}$. Note that we assume a purely resistive effect of the precipitate and not a capacitive one. This assumption is supported by the results of a d.c. measurement, as shown below.

Materials and methods

ISFETs with a $600 \AA$ thermally-grown $\mathrm{SiO}_{2}$ gate and $1500 \AA \mathrm{Ta}_{2} \mathrm{O}_{5}$ layer were used. The gate length $(L)$ and width $(W)$ were $15 \mu \mathrm{m}$ and 500 $\mu \mathrm{m}$, respectively. The ISFETs were encapsulated with Hysol epoxy after mounting the chips on a strip of printed circuit board. $5 \mu l$ human serum albumin (HSA; Sigma) in aqua dest $1.2 \mathrm{mg} / \mathrm{ml}$, was physically adsorbed on the gate of the ISFET chip. It was dried in a furnace at $37^{\circ} \mathrm{C}$ for three hours. Then an equivalent amount of anti-HSA (Dakopath Denmark) was added to the HSA and an insoluble immunological HSA-antiHSA complex was formed, which was visible as a white precipitate over the gate area. The immunological precipitate was dried at room temperature overnight and had a thickness of about $20 \mu \mathrm{m}$. The buffers used in the experiments were: $10^{-1}$, $10^{-2}$ and $10^{-3} \mathrm{M}$ TRIS/HCl buffers, $\mathrm{pH} 8.60$, and $10^{-1}, 10^{-2}$ and $10^{-3} \mathrm{M}$ HEPES/KOH buffer, $\mathrm{pH} 6.60$.

Two types of ISFET amplifiers were used. For stable d.c. measurements of the original and the immunomodified ISFETs, a source-drain follower circuit was used, as reported elsewhere [4]. We used a combined glass $\mathrm{pH}$ electrode (Amagruss) as reference and calibration electrode for the ISFETs. In this way the source voltage $\left(V_{\mathrm{g}}\right)$ as detected by the ISFETs could be 


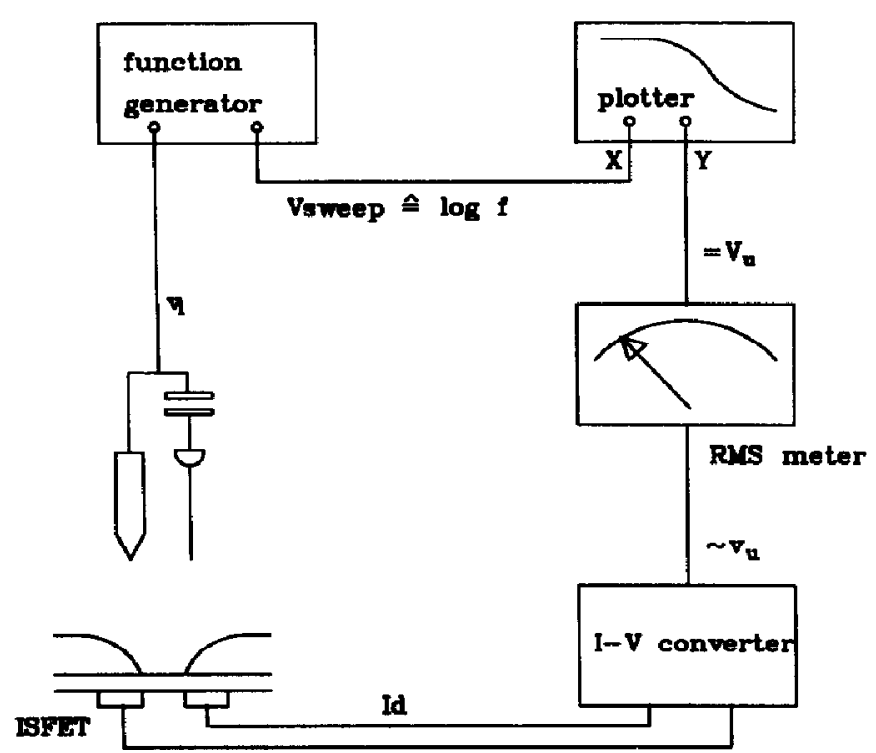

Fig. 3. Block diagram of the a.c. measuring system.

verified with the calibrated glass electrode. The solution was shielded from light and was stirred at low speed with a magnetic stirrer.

For the measurement of the frequency response of the ISFETs, an $I-V$ converter was used, as shown in Fig. 3. A $20 \mathrm{mV}$ (peak-peak) sinusoidal signal $\left(v_{1}\right)$ of frequency $f$ was applied to the calomel reference electrode. To decrease the reference electrode impedance, a Pt wire with a $1 \mu \mathrm{F}$ series capacitor was connected in parallel to the reference electrode. In this way a low value of $R_{\mathrm{el}}$ is provided for all frequencies. The ISFET was placed in a fixed position with respect to the reference electrodes. The amplification factor of the ISFET was normalized at $1 \mathrm{kHz}$ by adjusting the source-drain voltage. The amplitude of the output signal of the $I-V$ converter $\left(V_{u}\right)$ was measured with a r.m.s. a.c. voltmeter and applied to the $Y$ input of a $X Y$ recorder. $\log f$ from the function generator with automatic frequency sweep (Philips PM 5131) was connected on the $X$ input of the recorder. In our experiments the magnitude of the transfer function is sufficient to characterize the presence of the precipitate. The phase shift will not give additional information.

\section{Results}

\section{(a) D.c. behaviour}

An ISFET with precipitate and a bare ISFET were connected to the source-drain follower. The ISFETs were conditioned in $0.1 \mathrm{M}$ TRIS/HCl buffer, pH 8.60, for 20 minutes. We always found a large initial drift of the ISFET with immunological precipitate. A step in the $\mathrm{pH}$ value was carried out by adding an equal volume of $0.1 \mathrm{M}$ HEPES/KOH, $\mathrm{pH} 6.60$, resulting in a $\Delta \mathrm{pH}$ of one unit at constant buffer concentration. The experiments 


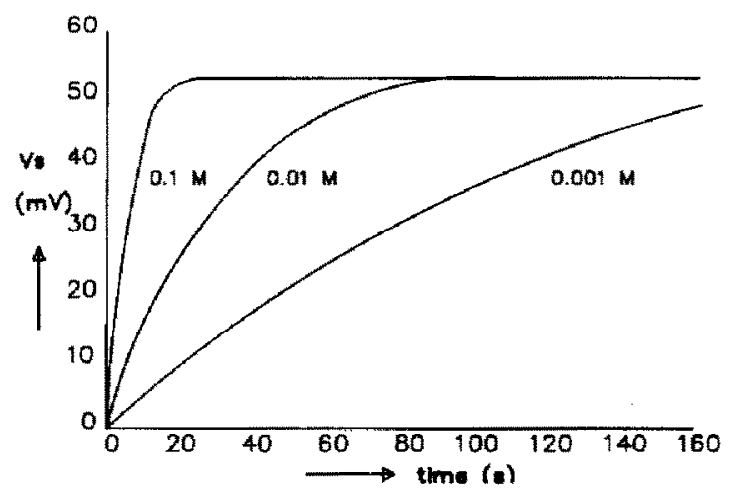

Fig. 4. pH-Step response of an ISFET with precipitate on $0.1 \mathrm{M}, 0.01 \mathrm{M}$ and $0.001 \mathrm{M}$ TRIS/HEPES buffers.

were repeated with $0.01 \mathrm{M}$ and $0.001 \mathrm{M}$ buffers. Figure 4 shows the observed responses on the $\mathrm{pH}$ step as a function of the buffer concentrations. When a thicker precipitate layer was present the time constants increased (results not shown). The response time of the bare ISFETs was independent of buffer concentration, typically $0.1 \mathrm{~s}$. The protein precipitate did not influence the pH sensitivity of the ISFET measured in the different buffers $(\sim 50 \mathrm{mV} / \mathrm{pH}$ unit). However, the response curves show clearly that a protein layer limits the diffusion of ions and influences the ISFET time behaviour, having a greater effect for lower buffer concentrations of the electrolyte [6].

\section{(b) A.c. behaviour}

The influence of the precipitate layer can be characterized in more detail by measuring the transfer characteristic of the ISFET with or without the protein precipitate. The qualitative model proposed in the previous Section may then be used to interpret the results.

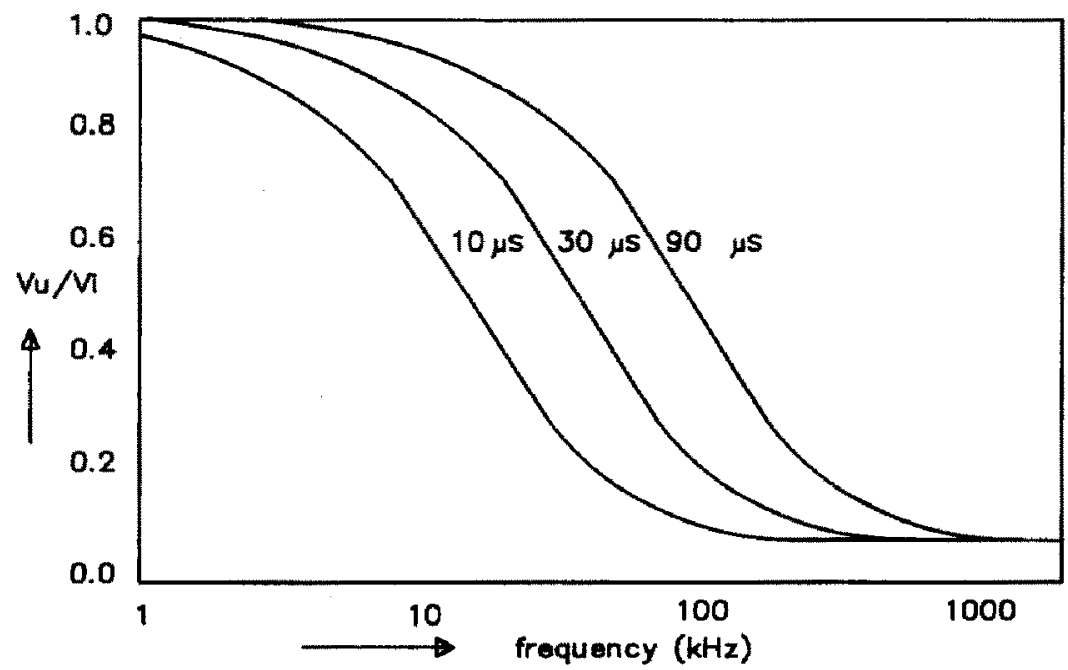

Fig. 5. Transfer characteristic of an ISFET, measured with the circuit shown in Fig. 3 for three different solution conductivities. 
Figure 5 shows the transfer characteristics of a bare ISFET at three different concentrations of the electrolyte, expressed in terms of specific conductivity in $\mu \mathrm{S}$. We found a linear relation between the specific conductivities of the different electrolyte concentrations and the measured cut-off frequencies. Good linearity was obtained up to frequencies of $500 \mathrm{kHz}$.

An ISFET with a precipitate was measured in a $10^{-3} \mathrm{M}$ TRIS/HCl $\mathrm{pH}$ 8.60 buffer with a specific conductivity of $30 \mu \mathrm{S}$. We observed a larger cutoff frequency $\left(f_{\mathrm{c}}\right)$ than with the bare ISFET at $30 \mu \mathrm{S}$; however, this cut-off frequency decreased as a function of time (Fig. 6). The amplification factor at $1 \mathrm{kHz}$ did not change. In the final state (curve 11), the value of $f_{\mathrm{c}}$ did not decrease any further, but remained larger than the value observed for the bare ISFET (curve 0). Probably at this time (after 12 minutes) an equilibrium with a specific value of $f_{\mathrm{c}}$ was established. The shift of $f_{\mathrm{c}}$ as a function of time is shown in Fig. 7.

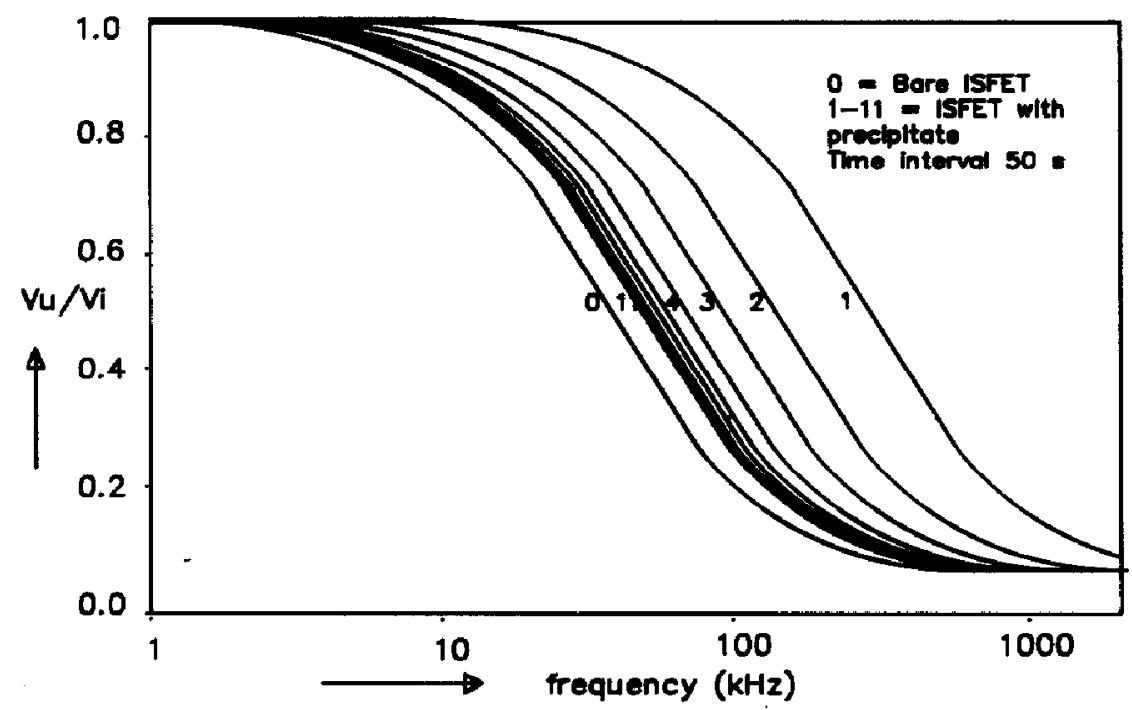

Fig. 6. Transfer characteristic of an ISFET with HSA-aHSA precipitate, measured with the circuit shown in Fig. 3.

The white immunological precipitate was later removed by cleaning the ISFET with a cotton wool stick without damaging the ISFET. The transfer function then shifted again (curve 0, Fig. 6). The final cut-off frequency was the same as observed with the original ISFET before the start of the experiments (curve $30 \mu \mathrm{S}$, Fig. 5).

We observed a pH-dependent and an ionic strength-dependent equilibrium value of $f_{\mathrm{c}}$. In Fig. $8 f_{\mathrm{c}}$ has been plotted as a function of $\mathrm{pH}$, showing a minimum around $\mathrm{pH}=6$ (buffer concentration $0.001 \mathrm{M}$ ). We found a smaller shift of the cut-off frequency equilibrium value with respect to the bare ISFET in the $0.01 \mathrm{M}$ buffers. 


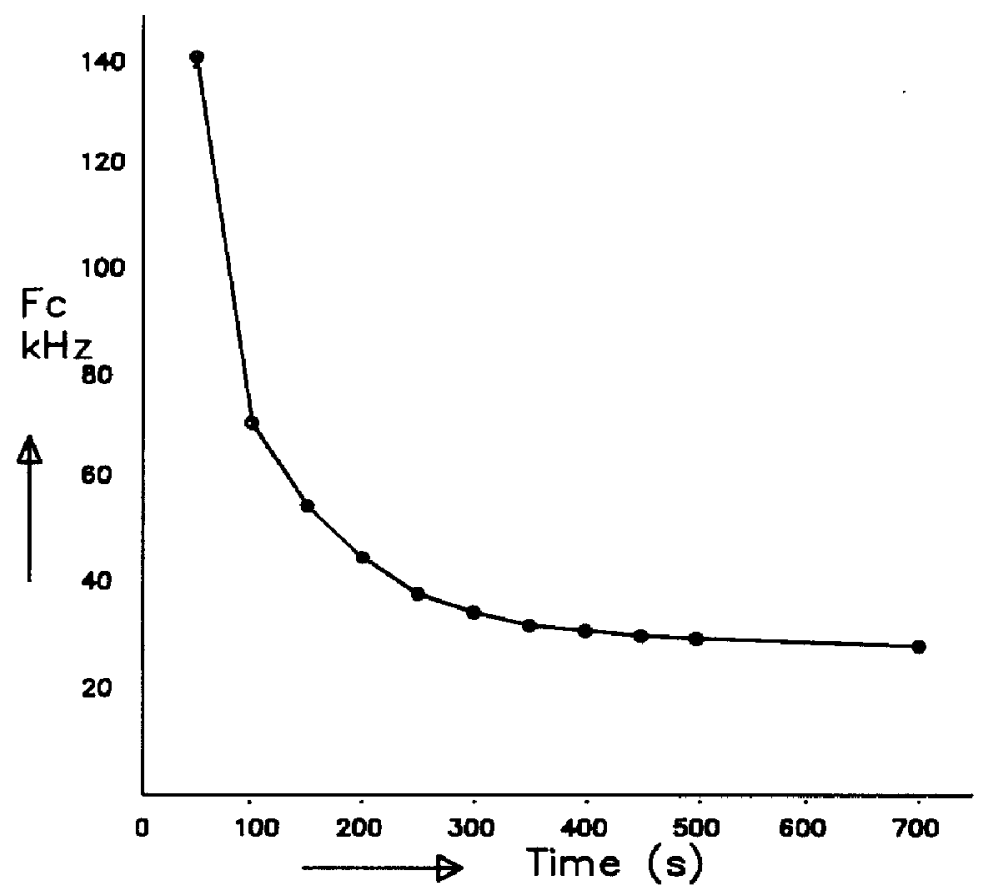

Fig. 7. Cut-off frequency $f_{c}$ as a function of time, derived from curves 1 to 11 of Fig. 6 .

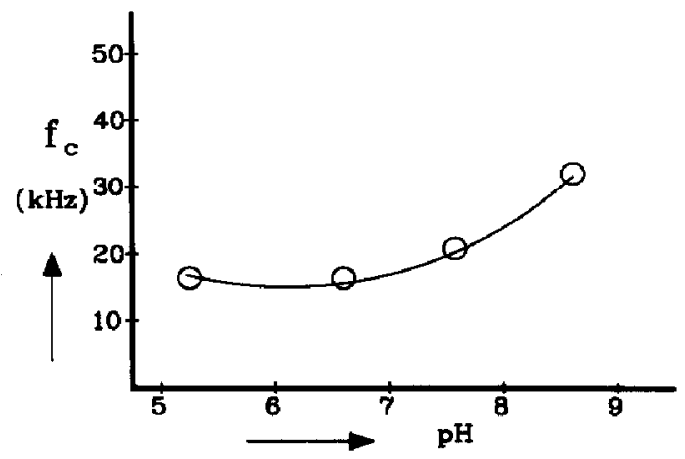

Fig. 8. Equilibrium value of the cut-off frequency $f_{\mathrm{c}}$ as a function of $\mathrm{pH}$ for $0.001 \mathrm{M}$ buffer solutions.

\section{Discussion}

The d.c. measurements show no measurable equilibrium gate-source voltage shift due to the presence of the immunological precipitate (Fig. 4). This may be the result of the large amount of counterions in the layer, screening every electrical charge. Also the pH sensitivity of the ISFETs is not affected by the precipitate, meaning that the layer is highly porous. This also means that the precipitate can be considered as a layer of electrolyte with a certain specific conductivity $\sigma_{p}$, as already mentioned in the theory, resulting in a purely resistive effect, as assumed in the qualitative model. It was found that the pH-step response time increased with de- 
creasing buffer concentrations (Fig. 4). This can be explained by assuming a relatively good buffering capacity of the protein complex at low electrolyte concentrations [5].

With respect to the a.c. measurements, Fig. 7 indicates that the ions present in the immunoprecipitate layer diffuse out of the layer after immersing the ISFET with precipitate into an aqueous solution. Since the largest contribution to the total resistance of the circuit as shown in Fig. 2(a) results from the layer closest to the surface of the gate area, the total resistance of the circuit will be influenced strongly by diffusion of ions out of the precipitate. As the result of this diffusion, the total resistance increased and the cut-off frequency decreased as a function of time (Figs. 6 and 7). However, at equilibrium there is still a higher conductivity in the protein complex than in the surrounding buffer, when a $10^{-3} \mathrm{M}$ TRIS/HCl buffer pH 8.6 is used.

This can be explained by the Donnan effect [6]. Immobile charges of the protein are screened by counterions. Because the charge density in an immunoprecipitate is larger than in the electrolyte, a redistribution of ions between buffer and precipitate will take place. According to the Donnan effect, the ionic strength of the solution as well as the charge density of the precipitate determines the ratio of the specific conductivities between the electrolyte and the protein complex. At high ionic strengths and $\mathrm{pH}$ values near the isoelectric point of the precipitate, the induced extra charge surrounding the protein molecules is relatively small and no shift of the equilibrium value of $f_{\mathrm{c}}$ will be observed with respect to the $f_{\mathrm{c}}$ value of a bare ISFET. This also explains the minimum of the $f_{\mathrm{c}}(\mathrm{pH})$ curve of Fig. 8 around pH 6.0, because the isoelectric point of the HSA-antiHSA protein complex is about 6.0. At lower pH values, the protein complex adsorbed on the ISFET was no longer stable.

If a capacitive effect of the protein precipitate existed, this should have resulted in an effective smaller input capacitance of the immunomodified ISFET. Then the amplification factor would have decreased. However, all measurements indicate a true resistive effect of the precipitate. The cut-off frequency always increases, initially as well as definitely, due to the presence of the protein precipitate. The capacitive effect of the protein can therefore be neglected, most probably due to the high ion concentration in the precipitate.

\section{Conclusions}

An immunoprecipitate on top of the gate of an ISFET does not change the d.c. behaviour, but changes the $\mathrm{pH}$ response time as observed by the time constants. The porous layer with charged macromolecules causes an ion redistribution between the electrolyte and the precipitate, which can be demonstrated by the frequency characteristics. At equilibrium an increased concentration of counterions exists in the immunoprecipitate, causing a 
decrease of the total resistance of the measuring circuit. As supported by the model, the protein layer resistance near the gate causes a decreased total resistance. The capacitive effect of the precipitate can be neglected. We observed that the thickness of the precipitate layer is an important factor in the final shift of the cut-off frequency. Because of the uncontrolled thicknesses of different protein layers with different crosslinking and swelling properties, the layers cannot be compared. An immunoprecipitate with a pH-dependent charge also has a pH-dependent resistance. Therefore different immunoprecipitates may be distinguished from each other by means of the isoelectric pH detected by the minimum shift of the cut-off frequency at equilibrium.

\section{Acknowledgement}

These investigations have been supported by the Foundation for Fundamental Research on Matter (FOM).

\section{References}

1 J. F. Schenck, Technical difficulties remaining to the application of ISFET devices, in P. W. Cheung (ed.), Theory, Design and Biomedical Applications of Solid State Chemical Sensors, CRC Press, Inc., Boca Raton, FL, 1978, pp. $165-173$.

2 J. Janata and R. J. Huber, Chemically sensitive field effect transistors, in H. Freiser (ed.), Ion Selective Electrodes in Analytical Chemistry, Vol. 2, Plenum Press, New York, 1980, p. 156.

3 J. Janata, Chemical selectivity of field effect transistors, Proc. 2nd Int. Meet. on Chem. Sensors, Bordeaux, France, July $7-10,1986$, pp. $25-31$,

4 P. Bergueld, The operation of an ISFET as an electronic device, Sensors and Actuators, 1 (1981) $17-29$.

5 G. S. Deem, N. J. Zabusky and H. Sternlicht, Association-dissociation time scale factor for proton transport in immobilized protein membranes, J. Membrane Sci., 4 (1978) $61-80$.

6 G. Stell and C. G. Joslin, The Donnan equilibrium: a theoretical study of the effects of interionic forces, Biophys. J., $50(1986) 855-859$.

\section{Biographies}

Richard Schasfoort was born in Oldenzaal, The Netherlands on April 3, 1959. He received his M.S. degree in chemical engineering, biotechnology at the State University, Groningen in 1984. In the same year, he started research on the development of immunological biosensors in the group of Prof. J. Greve, Applied Physics and Prof. P. Bergveld, Electrical Engineering at the Twente University of Technology, where he is now working towards his $\mathrm{Ph} . \mathrm{D}$. degree.

Geert Streekstra was born in Kampen, The Netherlands on November 13, 1961. He started his studies in applied physics in 1980 at the Twente 
University of Technology and as a student was involved in this research. He received the M.S. degree in applied physics in March, 1988.

Piet Bergueld was born in Oosterwolde, The Netherlands, on January 26,1940 . He received the M.S. degree in electrical engineering (electronics) from Eindhoven University of Technology, Eindhoven, The Netherlands, in 1965, and the Ph.D. degree from Twente University of Technology, Enschede, The Netherlands, in 1973.

Since 1965 he has bisen a member of the Bio-information Group, Department of Electrical Engineering, Twente University of Technology. $\mathrm{He}$ is further a member of the Coordination Centre for Biomedical Engineering and a member of the Research Unit Sensors and Actuators of this University. In 1981 he became a member of the Semiconductor Group of the Foundation for Fundamental Research on Matter in The Netherlands.

The subject of his dissertation was the ion-sensitive fieldeffect transistor (ISFET) and the OSFET. He is involved in research on electronic measuring and stimulating methods in physiological systems, with special attention on in vivo biosensors. He lectures on biomedical instrumentation for graduate students of Twente University of Technology and medical personnel of affiliated hospitals.

In 1984 he was appointed as full professor in biosensor technology at the Twente University of Technology.

Rob Kooyman received his M.S. degree in physics at Leiden University in 1975. From 1975 to 1980 he worked at the Agricultural University at Wageningen on a $\mathrm{Ph} . \mathrm{D}$. thesis devoted to molecular spectroscopy. At the University of Utrecht he developed spectroscopic methods for use in membrane biophysics. From 1985 he has been involved in biosensor research at the University of Twente.

Jan Greve was born in Koog aan de Zaan, The Netherlands, on October 24,1939 . He received his M.S. degree in experimental physics from The Free University, Amsterdam, The Netherlands, in 1967 and the Ph.D. degree from the same University in $\mathbf{1 9 7 2}$.

Since 1967 he has been working in the field of biophysics, applying and developing optical and spectroscopical techniques for the research of biological macromoles, bacteriophages and cells. In 1980 he started a biophysics subgroup of the optoelectronics group in the Applied Physics Department of the University of Twente. The current research interests of the group are micro-Raman spectroscopy of biological macromolecules, cell characterization, mini laser Doppler flowmeters and biosensors. 